IdeAs

Idées d'Amériques

15 | 2020

Eau et gestion de l'eau dans les Amériques

\title{
El agua en las Américas
}

\section{Vincent Dubreuil y François-Michel Le Tourneau}

Traductor. Marta Gómez

\section{CpenEdition}

\section{Journals}

Edición electrónica

URL: http://journals.openedition.org/ideas/8456

DOI: 10.4000/ideas.8456

ISSN: 1950-5701

Editor

Institut des Amériques

Referencia electrónica

Vincent Dubreuil y François-Michel Le Tourneau, «El agua en las Américas », IdeAs [En línea], 15 | 2020, Publicado el 01 marzo 2020, consultado el 23 septiembre 2020. URL : http:// journals.openedition.org/ideas/8456 ; DOI : https://doi.org/10.4000/ideas.8456

Este documento fue generado automáticamente el 23 septiembre 2020

\section{c) (i) $\Theta$}

IdeAs - Idées d'Amériques est mis à disposition selon les termes de la licence Creative Commons Attribution - Pas d'Utilisation Commerciale - Pas de Modification 4.0 International. 


\title{
El agua en las Américas
}

\author{
Vincent Dubreuil y François-Michel Le Tourneau \\ Tradución : Marta Gómez
}

1 La cuestión del agua es un tema recurrente en las Américas. El problema es aún más acuciante en el actual contexto de cambio climático global: la incierta evolución de la pluviometría en un clima cambiante, la creciente demanda por parte de una población cada vez más numerosa y el desarrollo de actividades agrícolas o industriales que consumen grandes cantidades de agua hacen que cada vez sea más complejo resolver la ecuación entre la oferta y la demanda. En las dos últimas décadas, la multiplicación de excesos en términos de déficit o de abundancia ha provocado una sucesión de «crisis del agua» que ha justificado una atención especial por parte de científicos en ciencias sociales y medioambientales.

2 A escala continental, los recursos hídricos son bastante abundantes. Las Américas representan por ejemplo el $45,5 \%$ del caudal fluvial total del planeta, pese a que el continente solo ocupa el 28,5\% de la superficie terrestre. Muchos ríos inmensos, como el Mississippi, el río San Lorenzo o el Orinoco, por no hablar del Amazonas, están fuera de categoría. El norte de América, en particular Canadá, cuenta además con un gran número de lagos que almacenan una parte significativa del agua dulce mundial y están cubiertos, temporal o permanentemente, de nieve o hielo, que también representan una parte importante de esta reserva. Sin embargo, este abundante recurso se caracteriza por una enorme disparidad en su distribución entre, por un lado, los desiertos de Atacama o de Mojave y muchas regiones áridas, como el noreste de Brasil o el suroeste de Estados Unidos, y, por otro, regiones superhúmedas como la Amazonia o las laderas del Pacífico de los Andes colombianos. El cambio climático afecta a esta distribución, pero más a menudo en el sentido de un aumento de las sequías en regiones ya de por sí poco húmedas que de una expansión de regiones bien irrigadas.

3 Esta desigual distribución se ve agravada por el «aprovechamiento» de unos recursos hídricos que son objeto de una intensa depredación desde Chile al suroeste de Estados Unidos (Texas, Arizona, California) o, desde un enfoque más comunitario, en los oasis andinos o nordestinos. Porque el agua es un recurso codiciado que permite el desarrollo de actividades económicas diversas: la agricultura -ya sea de regadío o no-, la 
industria y la energía, que son grandes consumidoras de agua, pero también los usos industriales y el consumo doméstico, que son principalmente urbanos y, por tanto, están cada vez más alejados de la fuente. Para abastecer a sus usuarios, existen enormes sistemas que desvían el caudal de ríos como el São Francisco o el Colorado hacia zonas áridas situadas a cientos de kilómetros de distancia, haciendo del agua una mercancía y un elemento transportado. Hablamos también un vector de transporte en sí mismo; ejemplo de ello son las vías fluviales de los ríos San Lorenzo y Paraná-Paraguay. El agua está, por tanto, en el centro de un universo americano marcado por una movilidad creciente.

4 En América, como en otras partes del mundo, el agua también es parte de una especie de vínculo social (o de su ausencia), tanto por la diversidad de prácticas que permite como por la representación que las sociedades se hacen de dicho recurso y por la forma en que lo integran en su desarrollo. En este sentido, la historia de la relación con el agua forma parte de la historia a secas. Desde la creación de la Autoridad del Valle del Tennessee (Tennessee Valley Authority), innumerables proyectos han tenido como objetivo la gestión integrada del agua. Desde los canales que atraviesan el desierto de Sonora o la Caatinga hasta las presas faraónicas de los ríos Columbia, Xingú o Iguazú, durante mucho tiempo ha prevalecido una lógica de planificación a gran escala. Así, se han contabilizado más de 12600 presas de gran envergadura en el continente americano ${ }^{1}$, además de doce de los treinta embalses más importantes y la mitad de las diez mayores centrales hidroeléctricas del mundo. Estos proyectos adquieren una complejidad adicional cuando los ríos o los acuíferos correspondientes atraviesan fronteras. Con todo, la dimensión continental de las cuencas hidrográficas implica negociaciones y relaciones de dominación entre Estados vecinos que abren una interesante vía a cuestiones geopolíticas clave.

5 Los desafíos, conflictos y representaciones relacionadas con el agua también se plantean a escala más local, y de forma exacerbada, en las metrópolis americanas. En primer lugar, a través de una dramática degradación de los recursos, hasta tal punto que a veces se ha llegado a escribir que las ciudades daban la espalda a sus lagos o a sus ríos. Cabe citar en este sentido el elevado nivel de contaminación del río Tieté -que se ha convertido en un verdadero problema para la ciudad de São Paulo- o cómo algunos ríos se han transformado en alcantarillas a cielo abierto en numerosas metrópolis, incluso de América del Norte, como Los Ángeles. Actualmente se observa un movimiento, iniciado en Estados Unidos, orientado hacia la reapropriación de las riberas de los ríos como parte de unas políticas municipales voluntaristas de «reconquista». Sin embargo, en muchas megalópolis latinas, la situación sigue siendo problemática, tanto por la contaminación de los cursos de agua como por la exposición de la población, a menudo la más humilde, a riesgos de inundación. La diversidad de situaciones americanas referidas al agua en las ciudades permite evaluar los conceptos de vulnerabilidad y resiliencia a nivel territorial.

6 Estos son los asuntos globales y locales que pretende abordar en toda su diversidad el presente número temático de la revista IdeAs dedicado al agua en las Américas. La dimensión medioambiental es fundamental: se pone el énfasis tanto en su componente físico (cuantificación de riesgos y recursos) como social y se toma explícitamente en consideración la complejidad del juego de actores territoriales a distintos niveles. En este sentido, son pertinentes los enfoques geográficos pero también históricos, 
políticos, sociales, económicos y culturales para contribuir a arrojar luz sobre un tema que abarca multitud de facetas.

7 Para este número, el comité editorial de la revista ha seleccionado ocho artículos. Los trabajos abarcan dos países de América del Norte (Estados Unidos y Canadá) y gran parte de América del Sur (Brasil, Chile, Argentina, Perú y Bolivia) y son representativos, además, de una gran diversidad de entornos: urbanos (Buckeye, Curitiba, São Paulo, Nuevo Brunswick), rurales (Región Nordeste, Atacama, Arizona), áridos, tropicales o fríos. La cuestión de los conflictos y los juegos de actores está presente en la mayoría de los estudios y afecta a categorías de población muy diversas entre las comunidades indígenas, los agricultores $\mathrm{y}$, por supuesto, los habitantes de las ciudades, cualquiera que sea su nivel y sus condiciones de vida: los autores plantean claramente la vulnerabilidad de cada uno de estos grupos y muestran la diversidad de situaciones locales, pero también el carácter recurrente de los problemas planteados por las inundaciones y el acceso a los recursos hídricos.

8 Un primer conjunto de textos aborda la cuestión del agua de forma global a través del estudio de las políticas, instituciones y programas implementados para gestionar los recursos hídricos y los conflictos relacionados con estos. El artículo de Florence Larocque (Who Fought for Water and What Did they Fight For? A Comparative Analysis of Water Conflicts in Latin America between 2000 and 2011) considera el agua como un objeto, a la vez único y multidimensional, de conflicto en Argentina, Chile, Bolivia y Perú: la dimensión comparativa de este texto permite esclarecer cómo y por qué los aspectos conflictivos del agua evolucionan con el tiempo y difieren de un país a otro. El texto de Carolina Milhorance, Daniela Nogueira y Priscylla Mendes (Do Programa Um Milhão de Cisternas ao Água para Todos: divergências políticas e bricolagem institucional na promoção do acesso à água no Semiárido brasileiro) aborda el caso de la Región Nordeste de Brasil, árida y semiárida, donde se han implementado los programas «Un millón de tanques de agua» y «Agua para Todos». Las autoras describen en detalle la dinámica de alianzas entre actores sociales, así como los conflictos con la burocracia estatal y las dificultades para poner en marcha políticas públicas a nivel local. En un contexto aún más árido, el del Atacama chileno, Hugo Romero y Manuel Méndez (Territorios hidrosociales en las geografías altoandinas del Norte de Chile: modernización y conflictos en la región de Tarapacá) analizan los procesos de producción discursiva y material de los territorios hidrosociales en el altiplano andino de la región de Tarapacá: según estos autores, los conflictos que cristalizan aquí en comunidades tradicionales están ligados a la introducción de concepciones occidentales del espacio, dentro del proceso de modernización de la región (irrigación, minas). El último texto, de Joan Cortinas y Franck Poupeau (Le champ des politiques hydriques dans l'Ouest étasunien: éléments d'interprétation des instruments d'action), desarrolla el estudio de las políticas hídricas frente a la sequía en el oeste de Estados Unidos: después de trazar la cartografía de las instituciones presentes en la cuenca del río Colorado, los autores proponen un análisis estadístico detallado y prestan una atención especial a los instrumentos de lo llamados water markets, cuyos usos varían de un Estado a otro.

El segundo grupo de textos se refiere de forma más concreta a los espacios urbanos, en los que la concentración de personas y actividades plantea cuestiones como los riesgos, la vulnerabilidad y los conflictos entre actores de una manera exacerbada. El texto de Guillaume Fortin, Charlotte Poirier, Francis Duhamel y Daniel Germain (Risques d'inondation et vulnérabilité: l'exemple du bassin versant de la rivière Kennebecasis, Nouveau- 
Brunswick, Canada) repasa los conceptos utilizados en ciencias sociales (riesgo, vulnerabilidad, amenaza, percepción) y su importancia para la adaptación de la población al cambio climático: dichos conceptos muestran cómo, a nivel de los barrios, la reducción del riesgo de inundación pasa por una mayor sensibilización de la población. Este tema del riesgo de inundaciones centra también el artículo de Gabriela Goudard y Francisco Mendonça (Eventos e episódios pluviais extremos: a configuração de riscos hidrometeorológicos em Curitiba, Paraná - Brasil). A través de la comparación de elementos físicos (precipitaciones) y de la vulnerabilidad de ciertas problaciones, los autores muestran cómo Curitiba, «ciudad modelo» desde el punto de vista medioambiental, se ha convertido también en una metrópolis expuesta a riesgos vinculados con episodios de lluvia extrema que, en ocasiones, se ven exacerbados por la falta de cooperación entre las comunidades de la aglomeración. En una ciudad aún más grande como São Paulo, Neli Aparecida de Mello-Théry, Hervé Théry, Danilo Pereira Sato y Heloisa de Camargo Tozato (Vulnérabilité, risques et conflits liés à l'eau : la zone de protection environnementale de la plaine inondable du Tietê) detallan, a través de un análisis del uso de la tierra, la vulnerabilidad y los riesgos de la llanura inundable del río Tieté y demuestran cómo presiones de todo tipo (demográficas, inmobiliarias, agrícolas e industriales), además del entramado de políticas y proyectos en una zona de protección medioambiental, han comprometido el funcionamiento de la llanura aluvial. Por último, el artículo de Anne-Lise Boyer y Rebecca F.A. Bernat (De la luzerne aux masterplanned communities : enjeux de la gestion de l'eau sur un front d'urbanisation, le cas de Buckeye en Arizona) expone las modalidades de gestión del agua a nivel municipal en Estados Unidos y muestra sus contradicciones: el laissez-faire a distintos niveles dentro del poder público (Estado, municipios) con respecto a la tierra limita la eficacia de los dispositivos creados para regular la explotación y la presión sobre los recursos hídricos.

\section{NOTAS}

1. Comisión Internacional de Grandes Represas (www.icold-cigb.org).

\section{AUTORES}

\section{VINCENT DUBREUIL}

Vincent Dubreuil est Professeur à l'Université Rennes 2 et membre de l'UMR 6554 LETG (Littoral Environnement Télédétection Géomatique). Géographe climatologue, ses travaux portent sur les risques (sécheresse) et les changements climatiques en France et au Brésil de l'échelle locale (îlots de chaleur urbain) à l'échelle régionale (impact de la déforestation en Amazonie sur le climat) et utilisent de manière privilégiée les données de satellites. Il coordonne ou participe à 
plusieurs projets de recherches en collaboration avec le Brésil (CNRS, ANR, COFECUB, H2020, FAPESP...) et a été professeur invité dans les universités de Brasilia, Presidente Prudente, Londrina et Fortaleza.

\section{FRANÇOIS-MICHEL LE TOURNEAU}

François-Michel Le Tourneau est directeur de recherche au CNRS et directeur-adhoint de l'UMI iGLOBES (CNRS-ENS-Université d'Arizona). 